\title{
SPACES CONGRUENT WITH BOUNDED SUBSETS OF THE LINE
}

\author{
GAIL S. YOUNG, JR.
}

This note is devoted to a characterization of bounded subsets of the line from the viewpoint of metric geometry. Such spaces have been characterized by Menger and others, ${ }^{1}$ usually from the consideration of conditions on imbeddability of finite subsets. The theorem given below differs from these in making use of a minimality property of the linear metric as the condition for congruence.

THEOREM. Let $S$ be a metric space with distance function $d(x y)$ and diameter $t$, possibly infinite. $A$ necessary and sufficient condition that $S$ be congruent with a bounded subset of the real line is that if $d^{\prime}(x y)$ is another metric for $S$ which is topologically equivalent ${ }^{2}$ to $d(x y)$ and in which $S$ has diameter $t$, then there are two points $a$ and $b$ of $S$ such that $d^{\prime}(a b)>d(a b)$.

Proof. The condition is necessary. For suppose that $S$ is a bounded subset of the real line with diameter $t$, and that there exists a metric $d^{\prime}(x y)$ for $S$ such that (1) for some two points $a$ and $b, d^{\prime}(a b)<d(a b)$, $d(x y)$ denoting the Euclidean metric for $S ;(2)$ for each two points $x$ and $y, d^{\prime}(x y) \leqq d(x y)$; and (3) there exist sequences $\left\{x_{n}\right\},\left\{y_{n}\right\}$, of points such that $d^{\prime}\left(x_{n} y_{n}\right)$ approaches $t$ as $n$ increases. From (2), $\lim d\left(x_{n} y_{n}\right)$ is also $t$. There is no loss of generality in assuming that

$$
\begin{aligned}
d\left(x_{n} y_{n}\right) & =d\left(x_{n} a\right)+d(a b)+d\left(b y_{n}\right) \\
& =d\left(x_{n} a\right)+d^{\prime}(a b)+d\left(b y_{n}\right)+\left[d(a b)-d^{\prime}(a b)\right] \\
& \geqq d^{\prime}\left(x_{n} a\right)+d^{\prime}(a b)+d^{\prime}\left(b y_{n}\right)+\left[d(a b)-d^{\prime}(a b)\right] \\
& \geqq d^{\prime}\left(x_{n} y_{n}\right)+\left[d(a b)-d^{\prime}(a b)\right] .
\end{aligned}
$$

By taking the limit of both sides of this last inequality, we get an immediate contradiction of (1).

The condition is sufficient. There exist sequences $\left\{x_{n}^{\prime}\right\},\left\{y_{n}^{\prime}\right\}$, of points of $S$ such that $\lim d\left(x_{n}^{\prime} y_{n}^{\prime}\right)=t$. Let $\left\{x_{n}\right\}$ be any subsequence of $\left\{x_{n}^{\prime}\right\}$. For each two points $x$ and $y$ let

Received by the editors May 6, 1946.

1 For definitions and bibliography, see L. M. Blumenthal, Distance geometries, University of Missouri Studies, 1938. This theorem had its beginning in discussions of some problems in measure with Dr. Dorothy Maharam.

2 That is, in order that $d\left(x_{n} x\right)$ approach zero, it is necessary and sufficient that $d^{\prime}\left(x_{n} x\right)$ approach zero. 


$$
h(x y)=\sup \left|d\left(x x_{n}\right)-d\left(y x_{n}\right)\right| .
$$

By the triangular inequality, for each $n$ both $d\left(x x_{n}\right)-d\left(y x_{n}\right)$ and $d\left(y x_{n}\right)-d\left(x x_{n}\right)$ are less than $d(x y)$, so that (5) $h(x y) \leqq d(x y)$. Clearly $h(x x)=0$ and $h(x y)=h(y x)$. Since, for each $n$,

$$
\left|d\left(x x_{n}\right)-d\left(z x_{n}\right)\right| \leqq\left|d\left(x x_{n}\right)-d\left(y x_{n}\right)\right|+\left|d\left(y x_{n}\right)-d\left(z x_{n}\right)\right|,
$$

we have

$$
h(x z) \leqq h(x y)+h(y z) .
$$

Now, let $\left\{y_{n}\right\}$ be the subsequence of $\left\{y_{n}^{\prime}\right\}$ corresponding to $\left\{x_{n}\right\}$. By (5), $h(x y) \leqq t$; on the other hand, $m$ being fixed, and $n$ varying,

$$
\sup \left|d\left(x_{m} x_{n}\right)-d\left(y_{m} x_{n}\right)\right| \geqq\left|d\left(x_{m} x_{m}\right)-d\left(x_{m} y_{m}\right)\right|=d\left(x_{m} y_{m}\right),
$$

so that (7) $\sup h(x y)=t$. Finally, from (5) we have that (8) a necessary condition that a sequence $\left\{a_{n}\right\}$ converge to a point $a$ is that $h\left(a_{n} a\right)$ approach zero.

It is not in general true that $h(x y)$ vanishes only if $x=y$, so that in general $h$ is not a metric. I shall, however, show that under our hypotheses it is.

Let $d^{\prime}(x y)=(1 / 2)[d(x y)+h(x y)]$. Using the properties (4)-(8) of $h$ and the corresponding properties for $d$, it is easily seen that $d^{\prime}$ is a metric equivalent to the metric $d$, that $d^{\prime}(x y) \leqq d(x y)$, and that sup $d^{\prime}(x y)=t$. It follows from the condition that $h(x y) \equiv d(x y)$, and hence that $h(x y)$ is independent of the particular subsequence of $\left\{x_{n}^{\prime}\right\}$ chosen.

This last statement implies that for each two points $x$ and $y$

$$
h(x y)=\lim \left|d\left(x x_{n}^{\prime}\right)-d\left(y x_{n}^{\prime}\right)\right|=d(x y) .
$$

For if this limit does not exist it is possible to find two subsequences $\left\{x_{1 n}\right\},\left\{x_{2 n}\right\}$, of $\left\{x_{n}^{\prime}\right\}$ such that for each $n$

$$
\left|d\left(x x_{1 n}\right)-d\left(y x_{1 n}\right)\right|<\left|d\left(x x_{2 n}\right)-d\left(y x_{2 n}\right)\right|
$$

and such that the upper limit of the left-hand side of (10) is less than the lower limit of the right-hand side. This implies that $h(x y)$ as defined by one of these sequences is less than $h(x y)$ as defined by the other. By considering the subsequences $\left\{x_{n}^{\prime} ; n>j\right\}$ for $j=1,2,3, \cdots$, it is easily seen that (9) holds.

Now let $a, b, c$ be any three points of $S$. By changing the lettering and taking a subsequence $\left\{x_{n}\right\}$ of $\left\{x_{n}^{\prime}\right\}$, we can assume that, for each $n$, 


$$
d\left(a x_{n}\right)-d\left(b x_{n}\right) \geqq 0 ; \quad d\left(b x_{n}\right)-d\left(c x_{n}\right) \geqq 0 .
$$

Adding the two members of (11) gives

$$
\left|d\left(a x_{n}\right)-d\left(c x_{n}\right)\right|=\left|d\left(a x_{n}\right)-d\left(b x_{n}\right)\right|+\left|d\left(b x_{n}\right)-d\left(c x_{n}\right)\right|,
$$

and letting $n$ increase in (12), we have by (9),

$$
d(a c)=d(a b)+d(b c) \text {. }
$$

Hence of any three points, one is between the other two, in the metric sense. By a well known result of Menger, ${ }^{3}$ if $S$ has more than four points it is congruent with a subset, $S^{\prime}$, of the line. If $S$ consists of exactly four points and is not a subset of the line, it is easy to reduce the distance between two of the points while leaving the diameter unchanged.

All that need be shown now is that $S^{\prime}$ is bounded. Suppose that it is not. We may assume that the origin belongs to $S^{\prime}$. Then the transformation sending the point of $S^{\prime}$ with abscissa $x$ into the point with abscissa $x / 2$ reduces each distance while leaving the diameter infinite, which contradicts our condition, and completes our proof.

Various special sets can be characterized by adding additional hypotheses to the condition. For example, an interval can be characterized as, say, a connected space in which the diameter is actually assumed by the distance between some two points, and which satisfies the condition of the theorem.

\section{Purdue University}

${ }^{3}$ Karl Menger, Untersuchungen über allgemeine Metrik, Math. Ann. vol. 100 (1928) pp. 75-163. See in particular p. 128. 